\title{
Autopercepción de competencias digitales en personal administrativo de la Red de Salud de Huánuco
}

\section{Self-perception of digital competences in administrative personnel of Huanuco health network}

\author{
Eler Borneo Cantalicio, 1,2,a \\ https://orcid.org/0000-0002-6273-9818
}

\section{Citar como}

Borneo Eler (2020). Autopercepción de competencias digitales en personal administrativo de la Red de Salud de Huánuco. Desafíos, 2020; 11(1); 19-24. doi: https://doi.org/10.37711/desafios.2020.11.1.148

\section{RESUMEN}

Objetivo. Determinar la percepción de los servidores públicos de una sede administrativa de salud sobre su grado de competencia digital. Métodos. Se llevó a cabo un estudio descriptivo con 87 servidores públicos (46 mujeres y 41 varones), procedentes de la sede administrativa de la Unidad Ejecutora 404 de la Red de Salud Huánuco, durante el 2020. En la recolección de datos se utilizó como instrumento una escala de competencia digital. Para el análisis inferencial se utilizó la prueba chi-cuadrado para una muestra. Resultados. Los hallazgos mostraron que el nivel general en competencia digital fue básico (62,1 \%). Específicamente, las dimensiones información y tecnología fueron percibidas en mayor porcentaje: 51,7 \% y $39,1 \%$, respectivamente; mientras que las dimensiones convivencia digital, comunicación y colaboración fueron percibidas en menor porcentaje. Y, estadísticamente se encontró mayor nivel de competencia digital en las competencias de nivel básico $(p \leq 0,000)$. Conclusión. El principal hallazgo muestra que los servidores públicos percibieron un nivel de competencia digital básico; es decir, el dominio de una sola dimensión de competencias.

Palabras clave: alfabetización digital, comunicación, convivencia digital, información.

\begin{abstract}
Objective. To determine public servants' perception from an administrative health branch on their degree of digital competence. Methods. A descriptive study was used. It was comprised of 87 public servants (46 women and 41 men), coming from the administrative branch of the Executing Unit 404 from the Huanuco Health Network, during 2020. For the data collection, a digital competence scale was used. For the inferential analysis, chi-square test was used for a sample. Results. Findings showed that the general level of digital competence was basic (62.1\%). Specifically, information and technology dimensions were perceived in a higher percentage: $51.7 \%$ and $39.1 \%$, respectively. Digital coexistence, communication and collaboration dimensions were perceived in a lower percentage. And, statistically, a higher level of digital competence was found in basic level skills $(p \leq 0.000)$. Conclusion. The main finding shows that public servants perceived a basic level of digital competence. In other words, the domain of only one dimension of competences.
\end{abstract}

Keywords: digital literacy, communication, digital coexistence, information.

2 Red de salud Huánuco. Perú

a Enfermero. Magister Salud Pública.
} 


\section{INTRODUCCIÓN}

Recientemente, el enorme desarrollo de internety de tecnologías de la información y de la comunicación (TIC) ha determinado una profunda transformación en el modo en que la sociedad se comunica, indaga información, produce conocimiento y lo comparte (Montero, Merino, Monte, Ávila y Cepeda, 2019).

Las TIC representan un pilar básico de la llamada economía digital que hoy en día es boyante. Su ininterrumpida evolución ha generado un contexto favorable para nuevos enfoques, en relación con los procesos de enseñanza-aprendizaje.

Además, el incremento de la incidencia tecnológica en la vida rutinaria, en global, y en los procesos de la administración pública, en particular, ameritan la adaptación y la modernización de estas como entidades y como organizaciones conformadas por personas. Para asegurar la prestación de servicios vinculados al gobierno y la participación electrónica, las entidades públicas necesitan conseguir competencias mediáticas que se relacionen a las competencias generales de la alfabetización digital e informacional impulsadas por la Organización de las Naciones Unidas para la Educación, la Ciencia y la Cultura y la Comisión Europea. Las mejoras en la alfabetización digital no deben valorarse únicamente dentro de las instituciones educativas, sino además requieren apropiarse en todas las entidades sociales, tanto públicas como privadas (Pérez-Tornero, GiraldoLuque, Tejedor-Calvo y Portalés-Oliva, 2018).

En el mismo sentido, Justicia y Pruna (2010) informan que ha cambiado el paradigma económico de industrial a informacional. Podríamos asegurar que el eficaz procesado de la información representa el objeto argumental de la competencia digital de los empleados en el ámbito público.

No obstante, en el entorno de los servicios sanitarios, esta transformación digital se está generando con más lentitud que en otros sectores; posiblemente en razón debido a la prudencia en la integración de la innovación que caracteriza a las instituciones, empresas o industrias en las que la vida de los pacientes puede estar en riesgo durante la prestación de los nuevos servicios (Berwick, 2003).

Desde el año 2005 la Organización Mundial de la Salud (2005) sugiere la integración a las políticas de salud de los países miembros, las distintas estrategias e infraestructuras de la salud digital, ya que estima que podrían alcanzar un impacto positivo a la hora de suministrar atención sanitaria a todo tipo de usuarios. Asimismo, podrían apoyar en otros espacios como la investigación o la salud pública.

Por otro lado, las competencias digitales han sido foco de investigaciones a partir del veloz crecimiento y apropiación de las TIC en la comunidad. De la misma forma como han ido evolucionando este tipo de tecnologías, el constructo inherente al despliegue de las habilidades, actitudes y conocimientos demandados en las personas ha sufrido adecuaciones (Torres-Gastelú, CorderoGuzmán, Soto-Ortíz y Mory-Alvarado, 2019).

La importancia de las competencias digitales se encuentra en su potencialidad para identificar un marco de trabajo que sitúa en interrelación directa los elementos pedagógico-didácticos de la enseñanza en el grado superior, y su pasaje y empleo efectivo en los contextos laborales de las diferentes disciplinas bajo espacios aceleradamente cambiantes y complejos.

Las competencias digitales tienen implicancias en el despliegue de un bagaje de habilidades, conocimientos y valoraciones que intervienen directamente sobre las innovaciones tecnológicas y sus distintas adopciones por parte de las diferentes disciplinas. Con lo cual, la medición de las competencias digitales en ambientes de aprendizaje viabilizaría el diseño y la ejecución de estrategias de enseñanza y aprendizaje de acuerdo al diagnóstico de situación (Fernández, Jofre, Fiotti y Odeon, 2019).

En el mismo sentido, múltiples organismos mundiales, institutos de investigación, universidades, investigadores/as y expertos/as han emprendido el estudio de las competencias digitales en tópicos que van desde su medición (Del Prado, 2015), generación de estrategias para su desarrollo (Zuñiga, 2016), directrices y estándares (ISTE, 2016), gestación de políticas públicas (Rovira, Santoleri y Stumpo, 2013), certificación y autoevaluación (Ferrari, 2013), hasta la reconceptualización (Ferrari 2013) que cubra las demandas de la sociedad del conocimiento.

El sector salud y sus trabajadores no debemos ser ajenos a este cambio, ya que la salud digital es una oportunidad para la mejora continua en espacios tan transversales como la innovación, la comunicación, la gestión por resultados, la gestión de información, la investigación, la docencia o la publicación científica (Montero, Merino, Monte, Ávila y Cepeda, 2019).

El proceso de cambio digital del sector salud está principalmente en función de la competencia de sus trabajadores. Es decir, por medio del desarrollo 
de competencias digitales entre los profesionales de la salud y de su evaluación se pueden diseñar las estrategias necesarias para disminuir la brecha existente y aligerar la transición digital del sector salud (Norman y Skinner, 2006).

Desde la Unión Europea se están promoviendo varias estrategias para disminuir esta brecha digital. Una de ellas es el Plan de Acción de Educación Digital (Comisión Europea, 2017), cuyo propósito principal es potenciar el despliegue de las competencias digitales necesarias para vivir y trabajar en una época de renovación digital. Otros proyectos europeos, como IC-Health, tienen como objetivo apoyar al desarrollo de la alfabetización digital de los trabajadores y usuarios europeos del sector salud a través de cursos virtuales masivos (IC-Health, 2018)

Por último, ante una realidad social y tecnológica tan cambiante como la que estamos viviendo, los trabajadores de la salud tenemos el deber ineludible de capacitarnos digitalmente en la mejora de nuestro desempeño profesional para así ofrecer una atención sanitaria cada vez mejor y adaptada al nuevo paradigma de alfabetización digital.

En ese sentido, el presente estudio tiene como objetivo determinar la percepción de los servidores públicos de una sede administrativa de salud sobre su grado de competencia digital, Huánuco al 2020.

\section{METODOS}

\section{Tipo de estudio}

Se trató de una investigación cuantitativa, observacional, prospectiva y transversal.

El diseño utilizado fue transversal.

\section{Población y muestra}

La población estuvo conformada por 87 servidores públicos de la sede administrativa de la Unidad Ejecutora 404 de la Red de Salud Huánuco, durante el año 2020. Se asumieron como criterios de inclusión: personal administrativo y de salud pública; y como criterios de exclusión: contratos eventuales, personal de servicios generales o con licencia por enfermedad.

\section{Recolección de datos}

La recolección de datos se efectuó entre los meses de abril y mayo de 2020, con la aplicación de una escala de competencia digital, tomando como referencia al cuestionario de Ambriz (2014), constituida por 33 preguntas de 5 alternativas: 1. Lo desconozco, 2. No podría realizarlo, 3. Sí, pero con ayuda, 4. Sí, siempre y 5. Sí y lo sabría explicar; y se distribuyeron en cuatro dimensiones: Información (7 îtems), Comunicación y Colaboración (10 ítems), Convivencia digital (6 îtems) y Tecnología (10 ítems). Se realizó la validación de contenido con la participación de cinco jueces expertos donde, por unanimidad, mostraron una la opinión favorable. La escala obtuvo una confiabilidad alta, de 0,946, según el coeficiente de alfa de Cronbach.

Asimismo, se realizaron las coordinaciones y capacitaciones oportunas para la recolección de datos mediante reuniones virtuales por Jitsi Meet. Posteriormente, se llevó a cabo la recolección de datos a través de la aplicación de la escala de competencia digital, cuyos îtems fueron respondidos por los servidores públicos.

\section{Análisis de datos}

Respecto al análisis descriptivo de los datos se utilizaron las medidas de frecuencia. En la comprobación de la hipótesis se utilizó la prueba de chi-cuadrado para la comparación de frecuencias de una sola muestra. Se tuvo en cuenta una significación de $\mathrm{P}<0,05$. $\mathrm{Y}$, para el procesamiento de los datos se utilizó el paquete estadístico IBM SPSS Statistics 22,0.

\section{RESULTADOS}

Dentro de las características generales encontramos que gran parte de los servidores públicos tenían edades entre 30 a 59 años, prevaleciendo el sexo femenino. Asimismo, prevalecía un nivel de estudios superiores, un tiempo de experiencia menor de 5 años y el desempeño de la función administrativa (ver tabla 1)

La dimensión con el mayor porcentaje de dominio fue la de Información. Un 51,7 \% indicó que podrían realizar tareas relacionadas con la planeación, búsqueda y selección de información en internet y, a partir de ella, elaborar un trabajo propio y además presentarlo usando algún software para elaborar mapas conceptuales, presentaciones, textos, vídeos o formato multimedia. La siguiente dimensión que más dominaron fue la Tecnológica, con un 39,1 \%. Los servidores públicos conocen y aplican los conceptos TIC básicos de las computadoras, su cuidado y resuelven problemas técnicos. También dominan las aplicaciones de uso extendido; es decir, utilizan funciones básicas de herramientas de productividad, tales como procesador de textos, hojas de cálculo, software para presentaciones, bases de datos, además de usar funciones básicas de herramientas de comunicación y colaboración 
Tabla 1.

Número de servidores públicos según características socioeconómicas. Red de Salud Huánuco, 2020

\begin{tabular}{lcc}
\hline Características generales & $\begin{array}{c}\text { Frecuencia } \\
(\mathrm{n}=87)\end{array}$ & \\
\hline & $\mathrm{fi}$ & $\%$ \\
Edad en años & 24 & 27,6 \\
21 a 29 & 61 & 70,1 \\
30 a 59 & 2 & 2,3 \\
60 a más & & \\
Género & 41 & 47,1 \\
Masculino & 46 & 52,9 \\
Femenino & & \\
Estudios & 14 & 16,1 \\
Técnica & 27 & 31,0 \\
Licenciatura & 22 & 25,3 \\
Especialidad & 24 & 27,6 \\
Maestría & & \\
Experiencia laboral en años & & 59,8 \\
Menor de 5 & 52 & 40,2 \\
5 a más & 35 & 29,9 \\
Función & & 70,1 \\
Salud Pública & 26 & \\
Administrativa & 61 & \\
\hline
\end{tabular}

a través de internet. Asimismo, el 24,1 \% dominaba la dimensión de convivencia digital y respecto a la dimensión de Comunicación y Colaboración. Finalmente, el $11,5 \%$ de los servidores públicos consultados, declaró dominar aspectos como el uso de protocolos sociales en ambiente digital (ver figura 1).

En general, el nivel de competencia que predominó en los servidores públicos fue el nivel básico, porque dominaron una sola dimensión de las cuatro que conformaban la competencia digital. En este sentido, 54 servidores públicos tuvieron un nivel básico; 29 servidores públicos declararon dominar dos o tres dimensiones, considerando con esto que tuvieron un nivel intermedio y 4 servidores públicos consultados tuvieron un nivel avanzado; dado que dominaron las cuatro dimensiones y se consideraron entonces competentes digitales (ver figura 2).

Por último, en cuanto al análisis inferencial de los resultados sobre el nivel de competencia digital se encontraron diferencias estadísticamente significativas con predominio del nivel básico ( $p \leq 0,000$ ); es decir el número de servidores públicos difiere dependiendo del nivel de competencia, siendo predominantes los servidores con un nivel básico de competencia digital (ver tabla 2).

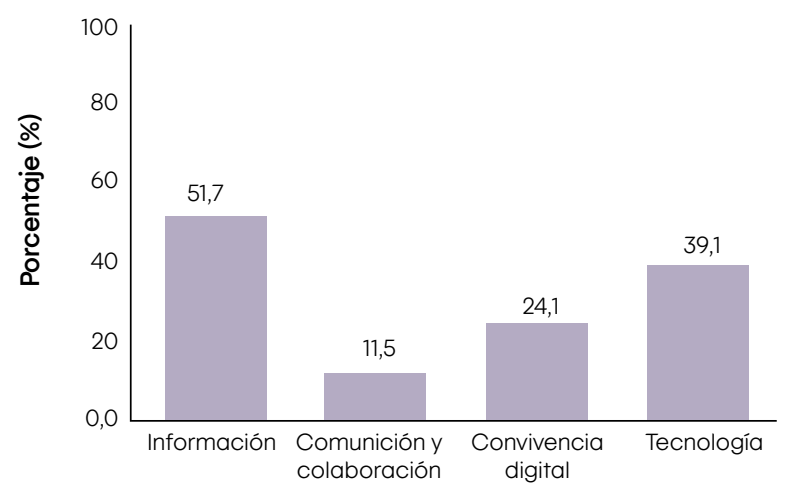

Figura 1. Número de servidores públicos según dimensiones sobre la competencia digital. Red de Salud Huánuco, 2020.

\section{DISCUSION}

En relación a los hallazgos referentes tenemos a Pérez et al. (2018) quienes mostraron que sólo el $26 \%$ de los trabajadores de entidades públicas encuestados realiza tareas creativas 0 de producción de contenido, el 50 \% no ha ejecutado nunca acciones colaborativas en internet y el $60 \%$ no lleva a cabo funciones vinculadas con el gobierno o la participación electrónica dentro de la administración pública.

Por otra parte, Jiménez-Pitre, Martel y Jaimes (2017) encontraron que los funcionarios encuestados se ubicaron en el nivel bajo sobre el empoderamiento digital; revelando que casi nunca emplean las ventajas y potencialidades de las TIC para atender los requerimientos de los ciudadanos en términos de productos y servicios que ofrecen las entidades públicas. Esto fue confirmado mediante los promedios resultantes para las dimensiones de accesibilidad, con un 2,5 y de integración digital, con un 2,5.

Asimismo, Martelo, Jiménez y Jaimes, (2017) hallaron bajos niveles de preparación personal, grado de preparación del personal de la empresa y

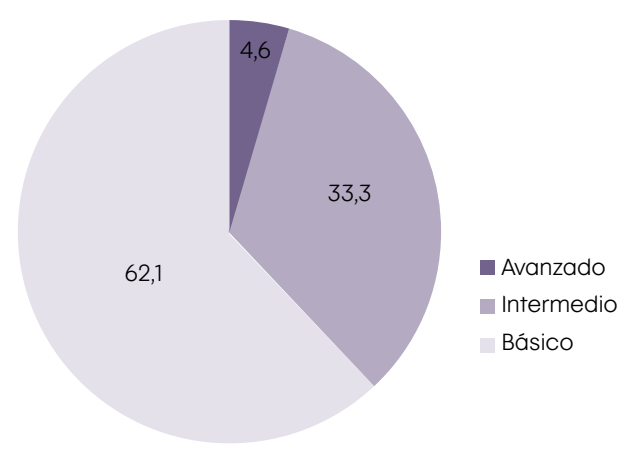

Figura 2. Nivel de competencia digital de los servidores públicos de la sede administrativa de la Unidad Ejecutora 404 de la Red de Salud Huánuco, 2020 
Tabla 2

Comparación del nivel de competencia digital de los servidores públicos de la sede administrativa de la Unidad Ejecutora 404 de la Red de Salud Huánuco, 2020

\begin{tabular}{lccccc}
\hline Nivel de competencia digital & $\begin{array}{c}\text { Frecuencia } \\
\text { observado }\end{array}$ & $\begin{array}{c}\text { Frecuencia } \\
\text { esperado }\end{array}$ & Residual & Prueba Chi cuadrado & Significancia \\
\hline Avanzado & 4 & 29 & -25 & \\
Intermedio & 29 & 29 & 0 & 43,10 & 0,000 \\
Básico & 54 & 29 & 25 & \\
Total & 87 & & & \\
\hline
\end{tabular}

empleo de las TIC; revelando, por consiguiente, los pocos esfuerzos ejecutados por los empleadores para lograr este tipo de formación, la insuficiente disposición de las MIPYME para la formación de sus trabajadores en competencias digitales y para generar mecanismos de accesibilidad e integración digital, tanto internamente como para sus usuarios a través del empleo de las TIC. Esta escasa disposición se evidenció también en el uso casi nulo de la página web para determinar relaciones de mercadeo, ventas y servicios al usuario; dado que no cuentan con las competencias digitales internas para su manejo y no poseen intenciones de solicitar asesoría externa para el empleo de este recurso tecnológico.

Igualmente, Domingo-Coscolla, Bosco, Carrasco y Sánchez (2020) concluyeron que el dominio de la competencia digital del docente universitario de idioma catalán (España) es deficiente. Habitualmente, menor en la competencia digital metodológica que en la competencia digital instrumental.

Por su parte, Castellanos, Sánchez y Calderero (2017), enfatizaron que, a pesar de vivir en la era de la información y la comunicación, el alumnado del grado universitario de Magisterio de Primaria no se encuentra lo suficientemente preparado en materia tecnológica para hacer frente a los requerimientos educativos que se les programa en su futuro cercano, donde han de producir, difundir y consumir cultura a través de las TIC.

Las implicancias del estudio están en función a que, según los resultados del mismo, podemos afirmar que los servidores administrativos del sector salud necesitan mayor capacitación e impulso en el dominio de las competencias digitales, a nivel intermedio o, en el mejor de los casos, a nivel avanzado; ya que la función pública en estos sectores es decisiva para la toma de decisiones a través de estas herramientas.

Finalmente, es necesario que las futuras investigaciones sobre este tópico aporten nuevos conocimientos sobre el impacto de factores estratégicos, normativos, organizativos y tecnológicos en la adopción de las nuevas tecnologías de información y comunicación en la provisión de servicios de salud pública.

En conclusión, se encontró que el número de servidores difiere dependiendo del nivel de competencia, siendo predominantes aquellos que presentan un nivel básico de competencia digital.

\section{REFERENCIAS BIBLIOGRAFICAS}

Ambriz, C. (2014). Competencia digital de los estudiantes. Estudio de caso: alumnos de nuevo ingreso a la Escuela Superior de Ingeniería Mecánica y Eléctrica Unidad Azcapotzalco (tesiss de maestría). Recuperado de https://tesis.ipn.mx/handle/123456789/14406

Berwick, D.M. (2003). Disseminating Innovations in Health Care. JAMA, 289(15): 1969-1975. doi: https://doi. org/10.1001/jama.289.15.1969.

Castellanos, A., Sánchez, C. y Calderero, J.F. (2017). Nuevos modelos tecnopedagógicos. Competencia digital de los alumnos universitarios. Revista electrónica de investigación educativa, 19(1), 1-9.

Comisión Europea. (2017). Plan de Acción de Educación Digital. Recuperado de https://ec.europa.eu/education/education-in-the-eu/digital-education-action-plan_en

De Prado, G. M. (2015) Competencia digital de los estudiantes que comienzan los estudios de Grado Maestro en Educación Primaria (Tesis Doctoral), Universidad de León, Léon, España.

Domingo-Coscolla, M., Bosco, A., Carrasco, S y Sánchez, J.A. (2020). Fomentando la competencia digital docente en la universidad: Percepción de estudiantes y docentes. Revista de Investigación Educativa, 38(1), 167-782. doi: http://dx.doi.org/10.6018/rie.340551

Fernández, D.C., Jofre, C.M., Fiotti, J. y Odeon, L. (2019). Proceso de definición de las competencias digitales para su evaluación en la educación superior en estudiantes de la carrera de psicología. En XI Congreso Internacional de Investigación y Práctica Profesional en Psicología. XXVI Jornadas de Investigación. $X V$ Encuentro de Investigadores en Psicología del MERCOSUR. I Encuentro de Investigación de Terapia Ocupacional. I Encuentro de Musicoterapia. Fa- 
cultad de Psicología - Universidad de Buenos Aires, Buenos Aires, Argentina.

Ferrari, A. (2013). DIGCOMP: Un marco para desarrollar y comprender la competencia digital en Europa. Sevilla: Institute for Prospective Technological Studies (IPTS), European Commission.

IC-Health (2018). Mejorando la alfabetización digital en salud de los ciudadanos europeos. Recuperado de https://ichealth.eu/.

ISTE (2016). ISTE standards students. Recuperado de https:// id.iste.org/docs/Standards-Resources/iste-standards_students-2016_one-sheet_final.pdf?sfvr$\mathrm{sn}=0.23432948779836327$

Jiménez-Pitre, I.A., Martel, R.J. y Jaimes, J. (2017). Escuela de Gobierno basada en TIC: Determinante para la Accesibilidad e Integralidad del Empoderamiento Digital. Información Tecnológica, 28(5), 75-86. doi: https://doi.org/10.4067/S0718-07642017000500010

Justicia, S. y Pruna, J. (2010). La competencia digital en el gobierno TI de las administraciones públicas. Tecnimap 2010. Recuperado den administracionelectronica.gob.es.

Martelo, R.J., Jiménez, I.A. y Jaimes, J. (2017). Accesibilidad e Integración Digital: Elementos Clave para un Programa de Formación de Empresarios en Empoderamiento Digital. Información Tecnológica, 28(6), 81-94. Recuperado de http://dx.doi.org/10.4067/ S0718-07642017000600010

Montero, J.A., Merino, F.J., Monte, E., Ávila, J.F. y Cepeda, J.M. (2019). Competencias digitales clave de los profesionales sanitarios. Educ Med. Recuperado de https://doi.org/10.1016/j.edumed.2019.02.010

Norman, C.D. y Skinner, H.A. (2006). eHEALS: Ia escala de alfabetización de eHealth. JMed Internet Res, 8(4): e27. doi: 10.2196/jmir.8.4.e27
Ocaña-Fernández, Y., Valenzuela-Fernández, L., Morillo-Flores, J. (2020). La competencia digital en el docente universitario. Propósitos y Representaciones, 8(1), e455. doi: http://revistas.usil.edu.pe/index.php/pyr/ article/view/455

Organización Mundial de la Salud. (2005). 58a Asamblea Mundial de la Salud: Ginebra, 16-25 de mayo de 2005: resoluciones y decisiones (Anexo). Recuperado de http://apps.who.int/iris/handle/10665/23058.

Pérez-Tornero, J.M., Giraldo-Luque, S., Tejedor-Calvo, S. y Portalés-Oliva, M. (2018). Propuesta de indicadores para evaluar las competencias de alfabetización mediática en las administraciones públicas. El profesional de la información, 27(3), 521-536.

Rovira, S., Santoleri, P., y Stumpo, G. (2013). Incorporación de TIC en el sector productivo: uso y desuso de las políticas públicas para favorecer su difusión. En S. Rovira y G. Stumpo (Ed.), Entre mitos y realidades. TIC, políticas públicas y desarrollo productivo en América Latina. Santiago: CEPAL. Recuperado de http:// repositorio.cepal.org/handle/11362/37255

Torres-Gastelú, C.A., Cordero-Guzmán, D.M., Soto-Ortíz, J.L. y Mory-Alvarado, A. (2019). Influencia de factores sobre la manifestación de la ciudadanía digital. Revista Prisma Social, 26, 27-49.

Zuñiga, J. I. (2016). Las competencias digitales en el perfil universitario: El caso de la Facultad de Pedagogía de la Universidad Veracruzana (Tesis Doctoral). Universidad Veracruzana, Veracruz, México.

Fuentes de financiamiento.

Autofinanciado.

\section{Correspondencia}

Eler Borneo Cantalicio Jr. San Luís Gonzaga n 104-Amarilis. Telf: 956419046

Email: eborneoc50@hotmail.com 